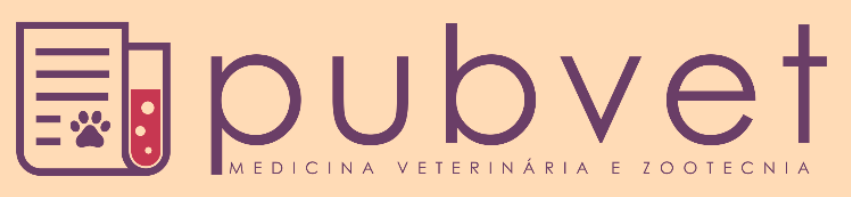

https://doi.org/10.31533/pubvet.v13n6a346.1-6

\title{
Dioctophyma renale: Revisão
}

\section{Milane Sales de Souza ${ }^{1 *}$, Grazielly Diniz Duarte ${ }^{2}$, Soraya Abrantes Pinto de Brito ${ }^{3}$ Leonardo Alves de Farias ${ }^{4} \bullet$}

${ }^{I}$ Graduando do Curso de Medicina Veterinária da UNINASSAU - João Pessoa-PB, E-mail: souzamilane@ hotmail.com; ${ }^{2}$ Graduando do Curso de Medicina Veterinária da UNINASSAU - João Pessoa-PB, E-mail: grazielly_ip@gmail.com;

${ }^{3}$ Graduando do Curso de Medicina Veterinária da UNINASSAU-João Pessoa-PB, E-mail: sorayabrito1970@ hotmail.com; ${ }^{4}$ Professor da Faculdade UNINASSAU - João Pessoa - PB. Brasil, E-mail: leovetleleco@gmail.com.

*Autor para correspondência

Resumo. O Dioctophyma renale é um nematoide, parasita animais domésticos e silvestres que causa a dioctofimose, designada como uma zoonose. Houve relatos raros da presença desse helminto em pele e rins de humanos. Tem maior incidência no Brasil, em cães não domiciliados e de hábitos alimentares pouco seletivos, mas há registros de casos de infecção no país em felinos e em espécies como cachorro do mato, lobo guará, quati e furão. O ciclo evolutivo é complexo e não totalmente elucidado, as infecções são normalmente assintomáticas e diagnosticadas por identificação de ovos em exames parasitológicos de urina e vermes por ocasião de cirurgias, necropsias, eliminações e algumas vezes após identificação por imagem. O tratamento mais eficaz é a remoção cirúrgica do parasito e a retirada do rim afetado, mesmo que a infecção ainda seja rara em algumas regiões, em outras ela ocorre em um crescente número, o que demonstra importância da realização de estudos, uma vez que, os dados da epidemiologia, ciclo biológico e diagnóstico do parasito ainda não apresenta registros claros, sendo em muitas ocasiões achados acidentalmente nas biópsias e cirurgias.

Palavras chave: cães, dioctofimose, parasitologia veterinária, zoonoses

\section{Dioctophyma renale: Review}

Abstract. Dioctophyma renale is a nematode, parasitic domestic and wild animal that causes diochophimosis, designated as a zoonosis. There have been rare reports of the presence of this helminth in human skin and kidneys. It has a higher incidence in Brazil, in non-domiciled dogs and in less selective eating habits, but there are records of cases of infection in the country in felines and in species such as wild dog, guará wolf, coati and ferret. The evolutionary cycle is complex and not fully elucidated; infections are usually asymptomatic and diagnosed by identification of eggs in parasitological exams of urine and worms during surgeries, necropsies, eliminations and sometimes after image identification. The most effective treatment is the surgical removal of the parasite and the removal of the affected kidney, although the infection is still rare in some regions, in others it occurs in a growing number, which shows the importance of carrying out studies, the epidemiological data, biological cycle and diagnosis of the parasite still do not present clear records, being in many occasions accidentally found in the biopsies and surgeries.

Keywords: dogs, diochophimosis, veterinary parasitology, zoonoses

\section{Dioctophyma renale: Revisión}

Resumen. El Dioctophyma renale es un nematodo, parásitos animales domésticos y silvestres que causa la dioctofimosis, designada como una zoonosis. Hubo relatos raros de 
la presencia de este helminto en piel y riñones de humanos. Em Brasil hay más incidencia de casos en perros callejeros y de hábitos alimenticios poco selectivos, pero hay registros de casos de infección en el país en felinos y en especies como el Perro de monte, Lobo Guará, Cuatí y Hurón. El ciclo evolutivo es complejo y no totalmente dilucidado, las infecciones son normalmente asintomáticas y diagnosticadas por identificación de huevos en exámenes parasitológicos de orina y gusanos en ocasión de cirugías, necropsias, eliminaciones y algunas veces después de identificación por imagen. El tratamiento más eficaz es la remoción quirúrgica del parásito y la retirada del riñón afectado, aunque la infección aún sea rara en algunas regiones, en otras se produce en un número creciente, lo que demuestra la importancia de la realización de estudios, ya que, los datos de la epidemiología, ciclo biológico y diagnóstico del parásito aún no presentan registros claros, siendo en muchas ocasiones hallados accidentalmente en las biopsias y cirugías.

Palabras clave: perros, dioctofimosis, parasitología veterinaria, zoonosis

\section{Introdução}

Dioctophyma renale é um nematódeo, pertencente a ordem Enoplidae, família Dioctophymetidae, superfamília Dioctophymatoidea, popularmente conhecido como verme gigante do rim, sua infecção é considerada uma zoonose (Andrade et al., 2010; Barriga, 2002; Lima et al., 2016; Mech \& Tracy, 2001; Monteiro, 2011; Monteiro et al., 2002; Silveira et al., 2015).

Embora a maior parte da infecção esteja associada às áreas rurais e tenha na fauna silvestre seus principais reservatórios, animais domésticos têm relevância na disseminação das doenças, e os animais de estimação uma importância ainda maior, sendo os cães e os gatos os que apresentam maior preocupação nesse sentido (Dyer, 1998; Ferreira et al., 2010).

As infecções geradas pelo verme são chamadas de Dioctofimose e normalmente nos cães são assintomáticos, os sinais clínicos quando presentes podem incluir fraqueza, dificuldade em caminhar, maior frequência de micção, anorexia, convulsões, ascite, cólicas abdominais, perda de peso, disúria e irritabilidade (Alvarenga et al., 1984; Alves et al., 2007; Ferreira et al., 2010; Silveira et al., 2015).

Objetivou-se realizar uma revisão de literatura sobre a Dioctophyma renale por um levantamento bibliográfico nas bases de dados Medline, Lilacs, Embase e ISI, relacionando os aspectos morfológicos gerais do parasito, ciclo evolutivo, espécies hospedeiras, localização do parasito, métodos diagnósticos, tratamento e a infecção gerada, para melhor compreensão e identificação do parasito e controle da zoonose.

\section{Morfologia}

O parasito D. renale possui como principais características morfológicas: tamanho grande, o macho podendo chegar a $45 \mathrm{~cm}$ de comprimento por 4 a $6 \mathrm{~mm}$ de largura, enquanto a fêmea podendo ter $100 \mathrm{~cm}$ de comprimento por $12 \mathrm{~mm}$ de largura. Apresenta uma coloração vermelho-escura conferida provavelmente pela hematofagia ao parasitar o hospedeiro definitivo. Possui uma boca pequena e simples, hexagonal e sem lábio, circundada por seis papilas dispostas em círculo. O macho possui na extremidade posterior uma bolsa copuladora onde emerge um único espículo e a fêmea apresenta órgão sexual simples na extremidade caudal obtusa (Alves et al., 2007; Fortes, 2004; Lima et al., 2016; Mech \& Tracy, 2001; Monteiro, 2011; Sousa et al., 2011; Taylor et al., 2017).

Os ovos são bioperculados, elípticos, coloração castanho-amarelada e apresentam casca espessa e enrugada, tampões bipolares com uma a duas células em seu interior. Após a eliminação na urina medem entre 73 a $83 \mu \mathrm{m}$ de comprimento e 45 a $47 \mu \mathrm{m}$ de largura (Lima et al., 2016; Monteiro, 2011; Pedrassani et al., 2009; Taylor et al., 2017).

\section{Espécies hospedeiras}

Os anelídeos oligoquetas parasitas das brânquias de crustáceos, rãs e peixes dulcícolas são os hospedeiros intermediários do D. renale. Os hospedeiros paratênicos incluem sapos (C. Ictericos), rãs (ranna spp.) e peixes de água doce, enquanto no grupo dos hospedeiros definitivos estão os: Mustelidae 
como vison, marta e lontra; Canidae como coiote, lobo, cão, lobo guará, cachorro do mato, raposa vermelha; Procyonidae como guaxinim e quati. Alguns autores citam ainda que, excepcionalmente, possam ser parasitados bovinos, suínos, gatos e humanos com menor intensidade (Brun et al., 1998; Fortes, 2004).

\section{Ciclo evolutivo}

O ciclo evolutivo é complexo, indireto e não totalmente elucidado, sendo os vermes ovíparos. Os ovos em estágio de célula única são excretados na urina morulados, na forma de agregados ou cadeias e no ambiente, a L1 se desenvolve dentro do ovo em torno de 35 dias. Os ovos são ingeridos pelo hospedeiro intermediário, no qual ocorrem duas mudas pré-parasitárias (L2 e L3). O período de desenvolvimento no hospedeiro intermediário é de aproximadamente de 2 a 4 meses. O hospedeiro final é infectado por meio de ingestão do hospedeiro intermediário junto com a água ou por meio da ingestão do hospedeiro paratênico que ingiram o anelídeo infectado com L3. No hospedeiro final, a larva infectante (L3) penetra na parede do intestino, alcança a cavidade peritoneal e, por fim, penetra no rim, podendo localizar-se em vários órgãos, onde passa a L4 e adultos. O período pré-patente é de, aproximadamente, seis meses, todavia, há relato de período tão longo quanto dois anos (Monteiro, 2011; Taylor et al., 2017).

\section{Localização}

O parasito é encontrado frequentemente no rim direito; porém, pode ser observado no rim esquerdo, cavidade abdominal, cavidade torácica, ureteres, bexiga, uretra, útero, ovário, glândula mamária, fígado, estômago e tecido subcutâneos de animais domésticos e silvestres (Monteiro, 2011; Pedrassani et al., 2009; Silveira et al., 2015). Em humanos, há relatos da presença do verme na pele e nos rins, ocasionando cólicas renais e hematúria (Oliveira et al., 2005; Sardjono et al., 2008).

\section{Sintomas e sinais clínicos}

A infecção gerada pelo helminto, geralmente acomete o rim do animal e a severidade da lesão depende do número de parasitas que afetam o rim, da duração da infecção, do número de rins envolvidos e da presença ou ausência de doença renal concomitante (Freitas et al., 2018; Kano et al., 2003; Monteiro et al., 2002; Sousa et al., 2011). Em geral os animais parasitados não apresentam sinais clínicos se nenhum ou pelo menos um rim for parasitado, geralmente o rim direito é acometido e há compensação pelo rim não afetado (Kano et al., 2003; Monteiro et al., 2002; Sousa et al., 2011). A maioria dos cães com parasitismo no rim apresenta acentuada destruição do parênquima. Diversos autores relataram destruição progressiva das camadas cortical e medular, reduzindo o órgão a uma capsula fibrosa (Ferreira et al., 2010; Kommers et al., 1999; Leite et al., 2005; Nakagawa et al., 2007).

A hidronefrose também é relatada e é causada pela obstrução do óstio uretral interno pelo nematóide adulto, que determina a atrofia com preservação do epitélio da pelve sendo considerada para o desenvolvimento do quadro clínico de insuficiência renal (Nakagawa et al., 2007).

Sinais como hematúria e dor renal são observados em decorrencia da destruição do parênquima renal. Observa-se ainda casos com desconfortos generalizados, relutância em caminhar, poliúria, polidpisia, anorexia, vômitos, aumento do volume da região iguinal, aumento de volume da região subcutânea em decorrência da migração errática do parasito, emagrecimento progressivo e aborto (Alvarenga et al., 1984; Alves et al., 2007; Silveira et al., 2015; Sousa et al., 2011). Há relatos com alto grau de parasitismo, em um só cão parasitado foram encontrados 34 exemplares de D. Renale, sendo 27 livres na cavidade abdominal e sete no interior do rim (Monteiro et al., 2002). Em estudo realizado em 28 cães em Uruguaina - RS observou-se a presença do parasito no rim direito em meio ao exsudato hemorrágico, parasitos livres na cavidade abdominal, no tecido subcutâneo na região inguinal e na região do flanco esquerdo, no interior de um cisto caudal ao rim direito e o número de parasitos variou de 1 a 24 exemplares, machos e fêmeas (Silveira et al., 2015). 


\section{Diagnóstico e tratamento}

O diagnóstico, em geral, até hoje é um achado acidental em necrópsias e durante cirurgias, embora a presença do helminto possa ser detectada pelo achado dos ovos operculados na urina, por radiografia e ultrasonografia dos rins e cavidade abdominal ou até mesmo, em casos raros, os vermes jovens podem ser eliminados pela urina (Barriga, 2002; Fortes, 2004; Leite et al., 2005; Monteiro, 2011; Monteiro et al., 2002; Pereira et al., 2008; Silveira et al., 2015; Soler et al., 2008; Roque et al., 2019; Venkatrajaiah et al., 2014; Vulcani et al., 2015).

No Brasil há relatos do parasito em cães (Kano et al., 2003; Kommers et al., 1999; Leite et al., 2005; Monteiro et al., 2002; Pereira et al., 2008; Silveira et al., 2015; Sousa et al., 2011; Roque et al., 2019), gatos (Verocai et al., 2009), espécies silvestres como cachorro-do-mato Cerdocyum thous (Ribeiro et al., 2009), lobo guará Chrysocyon brachyurus (Varzone et al., 2008; Vulcani et al., 2015), quati Nassua nassua (Milanelo et al., 2009), macaco Cebus apela (Ishizaki et al., 2010), furão Galictis cuja (Zabott et al., 2012) e também em humanos (Oliveira et al., 2005; Sardjono et al., 2008).

Atualmente não há opção farmacológica parasitária eficaz para o tratamento do D. Renale. Contudo, mesmo que o parasito perdesse sua viabilidade com o uso de anti-helminticos ele não seria facilmente removido do interior do rim devido a sua espessura e tamanho, sendo então a nefrectomia e a remoção cirúrgica do parasito o procedimento eletivo para o tratamento (Pedrassani et al., 2009). Um dos métodos de controle indicado na prevenção de Dioctofimose consiste em evitar que humanos e animais se alimentem de minhocas, rãs e peixes crus ou pouco cozidos especialmente em áreas endêmicas. Todavia, relata-se maior problemática, quando se trata de medidas de controle para animais domésticos de vida semidomiciliar, tornando a medida pouco eficaz (Ishizaki et al., 2010; Monteiro, 2011; Nakagawa et al., 2007).

\section{Conclusão}

Existe um grande número de parasitos já relatados em estudos no Brasil, apesar da maioria ser diagnosticado ocasionalmente em biópsias, cirurgias e necrópsias. Ressaltamos, portanto, a importância da realização de novos estudos envolvendo o $D$. renale, visto que, os dados da epidemiologia, ciclo biológico e diagnóstico do parasito ainda não apresentem registros claros podendo dificultar a prevenção e o controle da zoonose.

\section{Referências bibliográficas}

Alvarenga, J., Matera, J. M., Barros, P. S., Randi, R. E. \& Sterman, F. (1984). Dioctophyma renale in a dog. Modern Veterinary Practise, 65(2):125.

Alves, G. C., Silva, D. T. \& Neves, M. F. (2007). Dioctophyma renale: o parasito gigante do rim. Revista Científica Eletrônica, 4(8):1-10.

Andrade, E. C., Leite, I. C. G., Oliveira Rodrigues, V. \& Cesca, M. G. (2010). Parasitoses intestinais: uma revisão sobre os seus aspectos sociais, epidemiológicos, clínicos e terapêuticos. Revista de APS, 13(2):231-240.

Barriga, O. (2002). Las enfermedades parasitarias de los animales domésticos em la América Latina. Santiago, Chile: Editorial Germinal, Santiago.

Brun, R., Hecker, H. \& Lun, Z.-R. (1998). Trypanosoma evansi and T. equiperdum: distribution, biology, treatment and phylogenetic relationship (a review). Veterinary Parasitology, 79(2):95-107.

Dyer, N. W. (1998). Dioctophyma renale in ranch mink. Journal of Veterinary Diagnostic Investigation, 10(1):111-113.

Ferreira, V. L., Medeiros, F. P., July, J. R. \& Raso, T. F. (2010). Dioctophyma renale in a dog: clinical diagnosis and surgical treatment. Veterinary Parasitology, 16(1/2):151-155.

Fortes, E. (2004). Parasitologia veterinária (4 ed.). São Paulo: Editora ícone.

Freitas, D. M., Maria, B. P., Vasconcelos, B. M. A., Jorge, A. L. T. A., Teodoro, A. N., Leonel Alves, E. G. L. \& Rosado, I. R. (2018). Nefrectomia unilateral em um cão parasitado por Dioctophyma renale: Relato de caso. PUBVET, 12(9), a.178, 1-7. 
Ishizaki, M. N., Imbeloni, A. A., Muniz, J. A. P. C., Scalercio, S. R. R. A., Benigno, R. N. M., Pereira, W. L. A. \& Lacreta Junior, A. C. C. (2010). Dioctophyma renale (Goeze, 1782) in the abdominal cavity of a capuchin monkey (Cebus apella), Brazil. Veterinary Parasitology, 173(3-4):340-343.

Kano, F. S., Shimada, M. T., Suzuki, S. N., Osaki, S. C., Menarim, B. C., Ruthes, F. R. V. \& Laidane Filho, M. A. (2003). Ocorrência da dioctofimose em dois cães no município de Guarapuava-PR. Semina: Ciências Agrárias, 24(1):177-180.

Kommers, G. D., Ilha, M. R. S. \& Barros, C. S. L. (1999). Dioctofimose em cães\&58; 16 casos Dioctophymosis in dogs\&58; 16 cases. Ciência Rural, 29(3):517-522.

Leite, L. C., Círio, S. M., Diniz, J. M. F., Luz, E., Navarro-Silva, M. A., Silva, A. W. C., . . Veronesi, E. M. (2005). Lesões anatomopatológicas presentes na infecção por Dioctophyma renale (Goeze, 1782) em cães domésticos (Canis familiaris, Linnaeus, 1758). Archives of Veterinary Science, 10(1):95-101.

Lima, C. S., Murakami, V. \& Nakusu, C. C. T. (2016). Dioctophyme renale o verme gigante do rim: revisão de literatura. Revista Investigação, 15(4):37-41.

Mech, D. L. \& Tracy, S. P. (2001). Prevalence of giant kidney worm (Dioctophyma renale) in wild mink (Mustela vison) in Minnesota. The American Midland Naturalist, 145(1):206-209.

Milanelo, L., Moreira, M. B., Fitorra, L. S., Petri, B. S. S., Alves, M. \& Santos, A. d. C. (2009). Occurrence of parasitism by Dioctophyma renale in ring-tailed coatis (Nasua nasua) of the Tiete Ecological Park, São Paulo, Brazil. Pesquisa Veterinária Brasileira, 29(12):959-962.

Monteiro, S. G. (2011). Parasitologia na medicina veterinária (Vol. 1). São Paulo: Roca.

Monteiro, S. G., Sallis, E. S. V. \& Stainki, D. R. (2002). Infecção natural por trinta e quatro helmintos da espécie Dioctophyma renale (Goeze, 1782) em um cão. Revista da FZVA, 9(1):9.

Nakagawa, T. L. D. R., Bracarense, A. P. F. R. L., Reis, A. C. F., Yamamura, M. H. \& Headley, S. A. (2007). Giant kidney worm (Dioctophyma renale) infections in dogs from Northern Paraná, Brazil. Veterinary Parasitology, 145(3-4):366-370.

Oliveira, L. L., Attallah, F. A., Santos, C. L., Wakofs, T. N., Rodrigues, M. C. D. \& Santos, A. E. (2005). O uso da ultrassonografia para o diagnóstico de Dioctophyma renale em cão-relato de caso. Paper presented at the Annals V Conferência Sul-americana de Medicina Veterinária, Rio de Janeiro, Brasil.

Pedrassani, D., Hoppe, E. G. L., Avancini, N. \& Nascimento, A. A. (2009). Morphology of eggs of Dioctophyme renale Goeze, 1782 (Nematoda: Dioctophymatidae) and influences of temperature on development of first-stage larvae in the eggs. Revista Brasileira de Parasitologia Veterinária, 18(1):15-19.

Pereira, B. J., Holanda, C., Oliveira, D. C., Lopes, B. F. \& Costa, F. S. (2008). Diagnóstico ultrassonográfico de dioctofimose em cão-relato de caso. PUBVET, 2(Arti 455):1-6.

Ribeiro, C. T., Verocai, G. G. \& Tavares, L. E. R. (2009). Dioctophyme renale (Nematoda, Dioctophymatidae) infection in the crab-eating fox (Cerdocyon thous) from Brazil. Journal of Wildlife Diseases, 45(1):248-250.

Roque, C. C. T. A., Brito, C. R., Regina, M., Taboada, P. P., Gomes, A. R. A., Baldini, M., Alves, L. C. \& Taboada, L. O. (2019). Diagnóstico de Dioctophyma renale em um cão na baixada santista através da ultrassonografia abdominal. PUBVET, 13(1), a248, 1-6.

Sardjono, T. W., Purmono, B. B., Iskandar, A. \& Gunawan, A. (2008). Dioctophymatosis renalis in humans: first case report from Indonesia. Paper presented at the Congresso Tropical Medicine and Parasitologia.

Silveira, C. S., Diefenbach, A., Mistieri, M. L., Machado, I. R. L. \& Anjos, B. L. (2015). Dioctophyma renale em 28 cães: aspectos clinicopatológicos e ultrossonográficos. Pesquisa Veterinária Brasileira, 5(11):899-905.

Soler, M., Cardoso, L., Teixeira, M. \& Agut, A. (2008). Imaging diagnosis-Dioctophyma renale in a dog. Veterinary Radiology \& Ultrasound, 49(3):307-308.

Sousa, A. A. R., Sousa, A. A. S., Coelho, M. C. O. C., Quessada, A. M., Freitas, M. V. M. \& Moraes, R. F. N. (2011). Dioctofimose em cães. Acta Scientiae Veterinariae, 39(3):1-4. 
Taylor, M. A., Coop, R. L. \& Wall, R. L. (2017). Parasitologia Veterinária. Rio de Janeiro, Brasil: Guanabara Koogan.

Varzone, J. R. M., Aquino, L. P. C. T. \& Rodovalho, M. V. T. (2008). Achados macroscópicos de lesões resultantes do parasitismo por Dioctophyma renale em lobo-guará (Chrysocyon brachyurus)-relato de caso. Ensaios e Ciência: Ciências Biológicas, Agrárias e da Saúde, 12(2):171-178.

Venkatrajaiah, N., Kalbande, S. H., Rao, G. V., Reddy, V. C., Reddy, S. H., Rao, P. R., . . Keerthi, A. (2014). Dioctophymatosis renalis in humans: first case report from India. Journal of the Association of Physicians of India, 62(10):70-73.

Verocai, G. G., Measures, L. N., Azevedo, F. D., Correia, T. R., Fernandes, J. I. \& Scott, F. B. (2009). Dioctophyme renale (Goeze, 1782) in the abdominal cavity of a domestic cat from Brazil. Veterinary Parasitology, 161(3-4):342-344.

Vulcani, V. A. S., Franzo, V. S., Araújo, D. P., Vicentim, F. R., Costa, O. M., Rangel, A. S. \& Gomes, L. A. (2015). Dioctophyma renale em Lobo-Guará na região geoeconômica de Jataí, GO, Brasilrelato de caso. Revista Brasileira de Medicina Veterinária, 37(2):149-152.

Zabott, M. V., Pinto, S. B., Viott, A. M., Tostes, R. A., Bittencourt, L. H. F. B., Konell, A. L. \& Gruchouskei, L. (2012). Occurrence of Dioctophyma renale in Galictis cuja. Pesquisa Veterinária Brasileira, 32(8):786-788.

Recebido: 2 de maio, 2019.

Aprovado: 28 de maio, 2019.

Publicado: 25 de junho, 2019.

Licenciamento: Este artigo é publicado na modalidade Acesso Aberto sob a licença Creative Commons Atribuição 4.0 (CCBY 4.0), a qual permite uso irrestrito, distribuição, reprodução em qualquer meio, desde que o autor e a fonte sejam devidamente creditados. 